\title{
Neuropeptide $Y$ regulates proliferation and apoptosis in granulosa cells in a follicular stage-dependent manner
}

\author{
Yoko Urata ${ }^{1,2^{*}}$ (D), Reza Salehi ${ }^{1}$, Patricia D. A. Lima ${ }^{1,3}$, Yutaka Osuga $^{2}$ and Benjamin K. Tsang ${ }^{1}$
}

\begin{abstract}
Background: The complex regulatory mechanism involved in ovarian follicular development is not completely understood. Neuronal neuropeptide Y (NPY) is involved in the regulation of feeding behavior, energy homeostasis, and reproduction behavior, while its function in ovarian follicular development is not clear. The objective of this study was to investigate if and how NPY regulates follicle development in the ovary.

Methods: All experiments were performed using Sprague Dawley rats. To understand NPY expression pattern at different stages of follicular development, NPY content was assessed using immunohistochemistry in individual follicles. NPY and its receptors expression pattern were evaluated in granulosa cells isolated from preantral (PA), early antral (EA) and late antral follicles (LAF). The influence of NPY on granulosa cell proliferation and apoptosis were further assessed in vitro, using Ki67- and TUNEL-positivity assays. To investigate whether NPY induced-proliferation in EA granulosa cells is mediated through the activation of NPY receptor Y5 (NPY5R) and Mitogen-activated protein kinase (MEK) signal pathway, EA granulosa cells were treated with NPY5R antagonist (CGP71683) and MEK inhibitors (PD98059 and U0126), and Ki67-positive cells were assessed.
\end{abstract}

Results: NPY protein expression was follicular stage-dependent and cell type-specific. NPY signal intensity in EA was higher than those in PA and LAF. Antral granulosa cells showed the highest signal intensity compared to mural granulosa cells, cumulus cells and theca cells. Granulosa cells NPY protein content and mRNA abundance were higher in EA than in LAF. NPY receptor contents in granulosa cells were follicular stage-dependent. While NPY reduced apoptosis of EA granulosa cells, it increased the proliferation through NPY5R and MEK pathway. In contrast, in LAF granulosa cells, NPY reduced proliferation and increased the number of apoptotic cells, with no significant effects on PA granulosa cells.

Conclusion: This study is the first to evaluate the intraovarian role of NPY in granulosa cells at various stage of follicular development. These results indicate that NPY regulates granulosa cells proliferation and apoptosis in a follicular stage-dependent and autocrine manner. NPY may play a role in pathogenesis of ovarian follicular disorders.

Keywords: Neuropeptide Y, Folliculogenesis, Neuropeptide, Proliferation, Apoptosis

\footnotetext{
*Correspondence: uyoko-tky@umin.ac.jp

'Departments of Obstetrics \& Gynecology and Cellular \& Molecular Medicine, Interdisciplinary School of Health Sciences, University of Ottawa; Chronic Disease Program, Ottawa Hospital Research Institute, Critical Care Wing, 3rd floor, Room W3107, 501 Smyth Road, Ottawa, ON K1H 8L6, Canada

${ }^{2}$ Department of Obstetrics and Gynecology, the University of Tokyo, 7-3-1

Hongo, Bunkyo-ku, Tokyo 113-8655, Japan

Full list of author information is available at the end of the article
}

(c) The Author(s). 2020 Open Access This article is distributed under the terms of the Creative Commons Attribution 4.0 International License (http://creativecommons.org/licenses/by/4.0/), which permits unrestricted use, distribution, and reproduction in any medium, provided you give appropriate credit to the original author(s) and the source, provide a link to the Creative Commons license, and indicate if changes were made. The Creative Commons Public Domain Dedication waiver (http://creativecommons.org/publicdomain/zero/1.0/) applies to the data made available in this article, unless otherwise stated. 


\section{Background}

Neuropepetide Y (NPY), a 36 amino-acid neuropeptide, is abundantly expressed in central and peripheral nervous system [1]. Five mammalian NPY receptors (Y1, Y2, Y4, Y5 and y6) have been cloned. All of Y1, Y2, Y4 and Y5 receptors are coupled to an inhibitory $G$ protein, except the y6 receptor which is truncated in the most mammals including human, but is functional in mice [2]. Neuronal NPY is involved in the regulation of feeding behavior [3], energy homeostasis [4], memory retention [5] and reproductive behavior [6]. Additionally, it has been reported that NPY also regulates proliferation $[7,8]$, apoptosis [9], immune [10] and reproductive functions as it is expressed by leydig cells $[7,11]$. Moreover, NPY acts on the luteal vascular system regulating corpus luteum function [8]. However, it is not clear whether and how NPY regulates follicle development.

In the ovary, NPY is expressed in the stromal tissue of small antral follicles, granulosa cells of large antral follicles as well as in the nerves and vessels of the theca internal layer $[8,9]$. Jørgensen et al. reported that ovarian follicular fluid contains NPY [10]. Moreover, NPY regulates the luteal vascular system in the sheep and induces oxytocin secretion from the corpus luteum [8], stimulates luteal estradiol synthesis and granulosa cell progesterone secretion in vitro $[12,13]$. It induces Bcl-2-associated X (BAX) mRNA and decreases proliferating cell nuclear antigen (PCNA) mRNA in porcine luteinized granulosa cells [14]. However, the main ovarian source of NPY, and whether and how NPY regulates follicular growth are not known.

The objectives of this study were to investigate (a) whether the granulosa cell is the follicular source of NPY; (b) if and how NPY regulates granulosa cell proliferation and follicular atresia; (c) which of the NPY receptors mediate its actions on the granulosa cell proliferation and apoptosis; and (d) whether the above mechanisms are follicular stage-specific. We hypothesized that the pattern of NPY and its receptor expression can determine granulosa cell fate (proliferation vs apoptosis) and is follicular stagedependent. To test this hypothesis, we assessed the expression of NPY and its receptors in granulosa cells isolated at different ovarian follicular stages in vivo. We also investigated the influence of NPY on granulosa cell proliferation and apoptosis at different follicular stages of development in vitro, as well as the signaling pathway involved in NPYinduced proliferation.

\section{Results}

Ovarian NPY mRNA and protein content is cell type- and follicular stage-specific

To determine if the granulosa cell is the primary follicular source of NPY, and whether its expression is follicular stage-specific, NPY signal intensity and localization were assessed by immunofluorescence in 21-day-old Sprague Dawley (SD) rat ovaries. NPY signals were evident in granulosa cells in primordial (data not shown), preantral (PA), early antral (EA) and late antral follicles (LAF), but could not be demonstrated in atretic follicles due to nonspecific binding (data not shown). NPY signal intensity in EA (one or multiple small fluid-filled cavities) was significantly higher than those in LAF (one fluid-filled cavity) and PA $(p<0.05)$. There was no significant difference in its signal intensity between PA and LAF (Fig. 1b; $p>0.05$ ). To determine if its expression is cell type-specific, we assessed the signal intensities of cumulus (granulosa cells beside the oocyte, Fig. 1a, i), antral (granulosa cells beside the antrum, Fig. 1a, ii) and mural granulosa cells (granulosa cells beside the basal lamina, Fig. 1a, iii) and theca cells (Fig. 1)a, iv. Since antral granulosa cells had a higher NPY signal intensity than cumulus, mural granulosa and theca cells (Fig. 1c; $p<0.05$ ), we focused our subsequent experiments on NPY in antral granulosa cells from EA stage. These results suggest that granulosa cell is the main follicle source of NPY and its expression is follicular stage-dependent.

To further confirm if granulosa cells express NPY in a follicular stage-dependent manner, Npy mRNA abundance and protein content were assessed in granulosa cells isolated from different follicular stages following in vivo stimulation with equine Chorionic Gonadotropin (eCG) for 0 (PA), 24 (EA) or $48 \mathrm{~h}$ (LAF). Npy mRNA abundance in granulosa cells of EA was 1.8 times higher than that of LAF (Fig. 2b; $p<0.05$ ) and that of PA tended to be higher than that of LAF (Fig. 2 b; $p=0.17$ ). NPY protein content in granulosa cells of PA and EA were 2.7 and 2.3 times higher than that of LAF, respectively (Fig. 2a, c; $p<0.05$ ), suggesting that granulosa cell NPY may be more important for the development of PA and EA than of LAF.

\section{Expression of granulosa cell NPY receptor is subtype- specific and dependent on the stage of follicular development}

To determine whether granulosa cell NPY receptors are expressed in a follicular stage-dependent manner, the protein content of various NPY receptor subtypes in granulosa cells from different stages of follicular development were compared. NPY receptor Y1 (NPY1R) content in granulosa cells of PA was 2.6 times higher than those of LAF, while NPY receptor Y2 (NPY2R) content in granulosa cells was not significantly different among the follicular stages. NPY receptor Y4 (NPY4R) content in granulosa cells of PA was 2.3 and 2.7 times higher than those of EA and LAF, respectively. NPY receptor Y5 (NPY5R) content in granulosa cells of PA and EA was 2.0 times and 1.9 times higher than those of LAF, respectively (Fig. 3). These results indicate that the expression of NPY receptor is subtype-specific and may be involved in the regulation of granulosa cell functions in a follicular stage-dependent manner. 


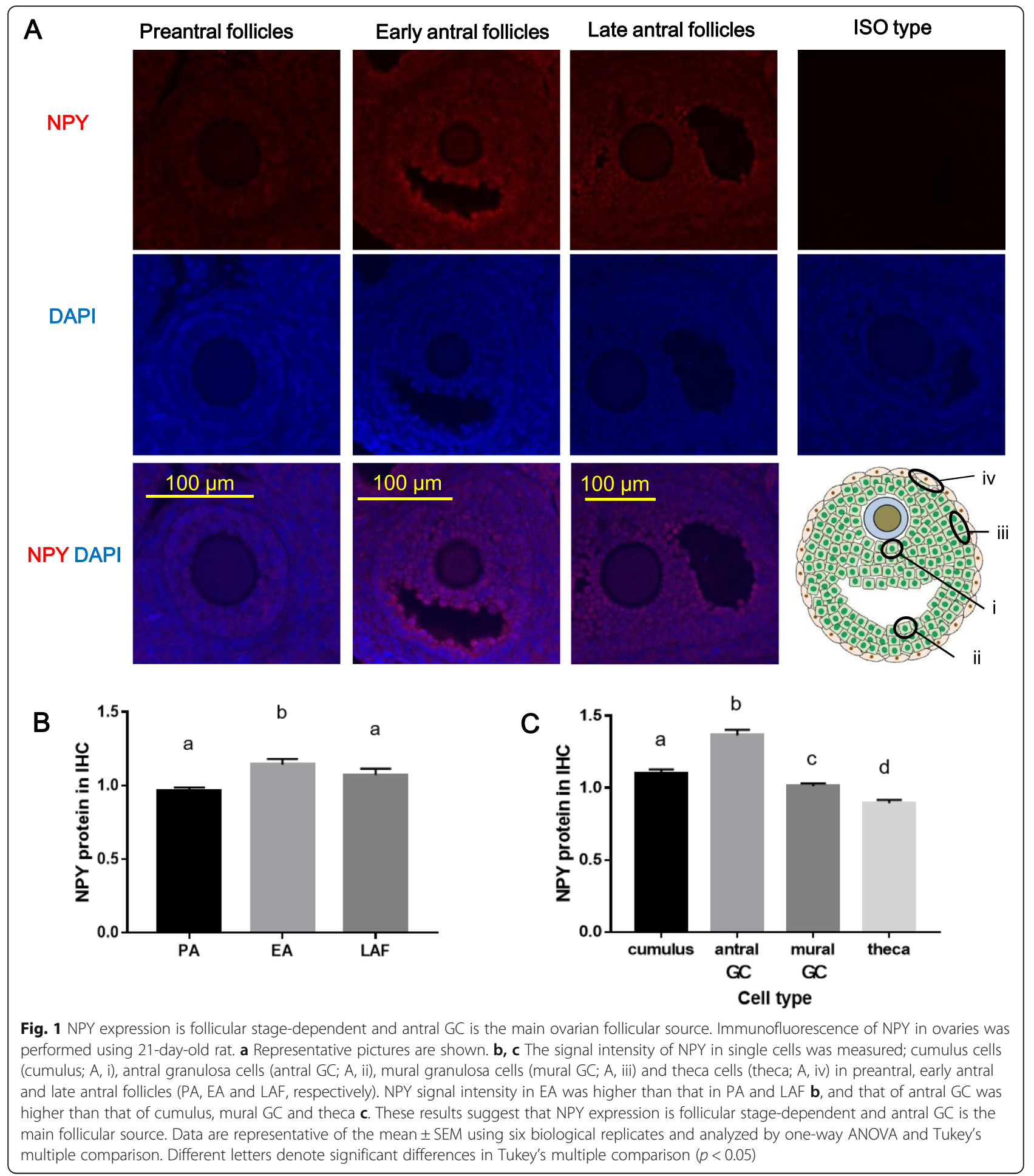

NPY induces proliferation in granulosa cells from early antral follicles and apoptosis from late antral follicles To investigate if NPY regulates granulosa cell fate (proliferation and apoptosis) in a follicular-stage dependent manner, granulosa cells were isolated from ovarian follicles in different stages of development and they were cultured in the presence of NPY at various concentrations $(0,0.001,0.1$ and $10 \mathrm{nM})$. To determine the rate of proliferation and apoptosis, Ki67- and TUNEL-positivity were assessed, respectively. Our results indicate that NPY at $0.1 \mathrm{nM}$ significantly increases Ki67-positivity in granulosa cells of EA by $11 \%(p<0.05)$, while reduces 


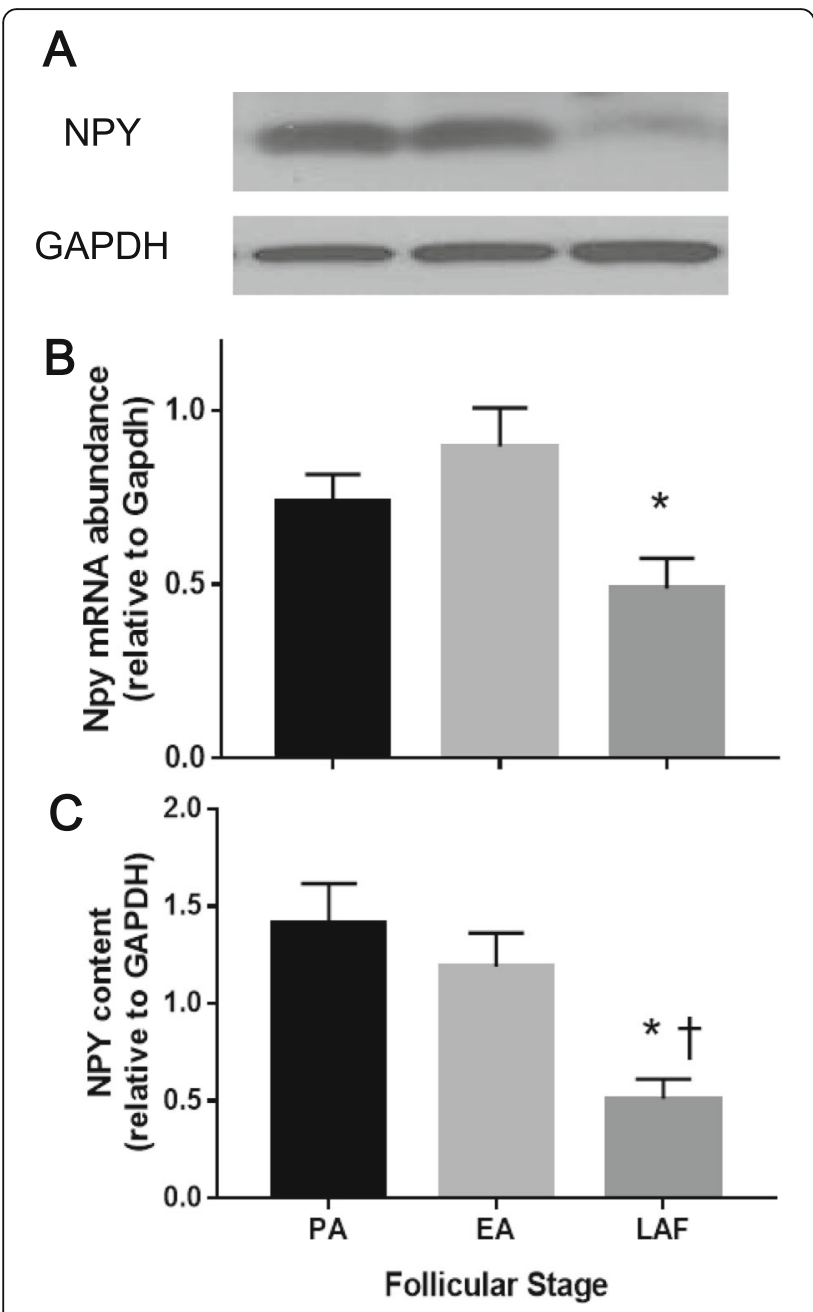

Fig. 2 NPY mRNA abundance and protein content of granulosa cells is higher in EA than LAF. Granulosa cells of PA, EA and LAF were isolated separately using rats primed with $\mathrm{ECG}$, as described in the methods section. a, c Total protein was extracted and NPY contents were assessed by Western blot. Representative Western blot result is shown a. NPY protein content of granulosa cells in PA and EA was higher than that in LAF $\mathbf{c}$. $\mathbf{b}$ Total mRNA was extracted, reverse transcribed and amplified by real-time PCR using specific primers for Npy and Gapdh. Expression of Npy mRNA was normalized to RNA loading for each sample using Gapdh mRNA as an internal standard. Npy mRNA of granulosa cells in EA was higher than that in LAF. These results suggest that granulosa cell NPY is more important for pre-antral and early-antral follicles development than at later stages. Each granulosa cell replicate was isolated from at least two rats. Results are expressed as mean \pm SEM of independent replicates ( $A$, $n=4 ; B, n=5)$ and analyzed by one-way ANOVA and Tukey's multiple comparison. *,$p<0.05$ (vs. EA); $\uparrow, p<0.05$ (vs. PA)

those of LAF by $15.7,18.2,14.5 \%$ at $0.001,0.1,10 \mathrm{nM}$, respectively (Fig. 4a; $p<0.05$ ). In contrast, NPY at 0.1 $\mathrm{nM}$ increases TUNEL-positivity in granulosa cells of LAF by $42 \%$ (Fig. 4 b; $p<0.05$ ). Additionally, NPY $(0.1$ $\mathrm{nM}$ ) increased the number of BrdU-positive granulosa cells of early antral granulosa cells by 1.2-folds (Fig. 4c; $p<0.05$ ). Our results indicate that NPY regulates granulosa cell fate in a follicular stage-dependent manner, inducing granulosa cell proliferation in early antral stage, but apoptosis in late antral stage.

\section{NPY induces granulosa cell proliferation through the activation of the NPY5R and via the mitogen-activated protein kinase pathway}

Since NPY5R contents of granulosa cells in EA was higher than that in LAF, we investigated whether NPY inducedproliferation is mediated through NPY5R activation by examining the influence of the NPY5R antagonist CGP71683 on NPY-induced granulosa cell proliferation. The NPY5R antagonist $(1 \mu \mathrm{M})$ significantly inhibited NPYinduced-Ki67 positivity in granulosa cells of EA (Fig. 5a; $p<0.05)$. In the absence of exogenous NPY, CGP71683 $(0.5,1 \mu \mathrm{M})$ had no significant effects on Ki67-positivity and trypan blue positivity (cell viability marker; data not shown), suggesting that the effect of the antagonist was specific and not cytotoxic.

To determine whether NPY-induced granulosa cell proliferation in EA is mediated through the Mitogenactivated protein kinase (MEK) signal pathway, granulosa cells were pre-treated for $1 \mathrm{~h}$ separately with the MEK inhibitors, PD98059 and U0126, and proliferation were assessed. Both of MEK inhibitors, PD98059 $(20 \mu \mathrm{M})$ and $\mathrm{U} 0126(2.5$ and $5 \mu \mathrm{M})$, significantly attenuated NPY-induced granulosa cell proliferation (Fig. 5b \& c; $p<0.05)$. In the absence of NPY, PD98059 and U0126 had no significant effect on Ki67-positivity (Fig. $5 \mathrm{~b} \& \mathrm{c}$ ) either trypan blue positivity (data not shown). These results indicate NPY regulates early antral granulosa cell proliferation through NPY5R and MEK signaling pathway.

\section{Discussion}

The molecular and cellular regulation of folliculogenesis is complex and not fully understood. NPY is known to regulate female reproductive function through the central neuron system [6]; NPY at the hypothalamus modulates the activity of GnRH neuronal system via NPY1R and NPY2R and regulates LH secretion by the pituitary gland [15-21]. However, its role in the control of the follicular development at the ovarian level has not been reported. In the present studies, we have demonstrated that: a) the expression of NPY and its receptors is follicular stage-dependent and cell type-specific; b) the influence of NPY on granulosa cell proliferation is different between EA and LAF; and c) NPY-induced granulosa cell proliferation was sensitive to NPY5R antagonist and MEK inhibitors, suggesting that NPY5R signaling may be an intra-ovarian regulatory event in folliculogenesis. These findings support the hypothesis that NPY promotes follicular survival and growth in EA but induces follicular atresia in LAF. 

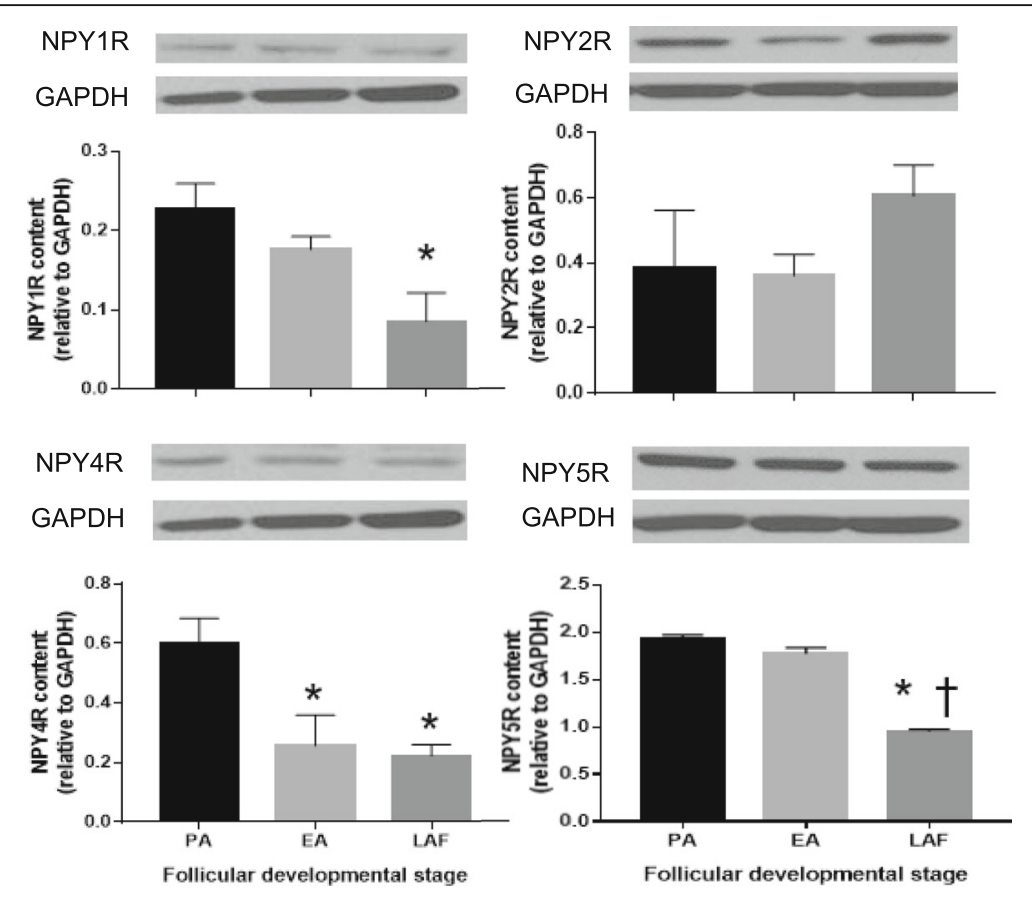

Fig. 3 NPY receptor Y5 content of granulosa cells in EA is higher than that in LAF. Granulosa cells of PA, EA and LAF were isolated separately from rats primed with eCG, as described in the methods section. Total protein was extracted and NPY receptors contents were assessed by Western blot $(n=3)$. Representative Western blot results are shown. NPY receptor Y1 (NPY1R) content of granulosa cells in PA was higher than that in LAF. NPY receptor Y2 (NPY2R) contents of granulosa cells showed no significant difference. NPY receptor Y4 (NPY4R) content of granulosa cells in PA was higher than that of EA and LAF. NPY receptor Y5 (NPY5R) content of granulosa cells in PA and EA was higher than that in LAF. These results suggest that expression of NPY receptor is subtype-specific and may be involved in the regulation of granulosa cell functions in a follicular stage-dependent manner. Each granulosa cell replicate was isolated from at least two rats. Results are expressed as mean \pm SEM of three independent replicates and analyzed by one-way ANOVA and Tukey's multiple comparison. ${ }^{*}, p<0.05$ (vs. PA);,$p<0.05$ (vs. EA)

The expression of NPY and its receptors NPY2R in the ovary has been reported $[8,22]$. However, whether these responses vary among follicular stages and if granulosa cells are the predominant source of follicular NPY remain to be determined. Our immunofluorescence results suggest that antral granulosa cells are the main follicle source of NPY with the highest expression in EA compared to LAF. However, our western blotting results show no difference of cellular NPY abundance between $\mathrm{PA}$ and EA. The reason for this apparent dichotomy is not known, but could be technical differences including purity of the isolated cells (e.g. mixture of antral- and mural-granulosa cells) and assay sensitivity (real-time PCR and Western blot). Regarding NPY receptors, we have found that granulosa cells express all NPY receptors, but their cellular levels vary among follicular stages. In contrast to NPY1R, NPY2R and NPY4R receptors, NPY5R is significantly higher in EA granulosa cells compared to LAF. Our results indicate that function of NPY/ NPY5R in granulosa cells varies among follicular stage and its response is strong at early antral stage.

Conflicting findings exist regarding the role of NPY in the regulation of cell proliferation; whereas NPY promotes proliferation in vascular smooth muscle cells [23], bone marrow stromal cells [24] and adipocyte precursor cells [7], it suppresses proliferation in luteinized porcine granulosa cells [14]. Moreover, whether the regulation of granulosa cell proliferation by NPY is follicular stagedependent is not known. In the present studies, we have demonstrated that NPY at physiological concentration $(0.1 \mathrm{nM})[25,26]$ induces proliferation in granulosa cells from EA, but not LAF in vitro. Additionally, an antagonist of NPY5R blocked NPY-induced granulosa cell proliferation in EA, as also demonstrated in neuroblastoma [27] and breast cancer [28, 29] cells. Although granulosa cells of PA and EA contain similar NPY5R protein contents, their responses are different. It is indicated that other NPY receptors may have the complementary or compensatory mechanism. Our results suggest that NPY/NPY5R regulates granulosa cell proliferation in a follicular stage-dependent manner, with an induction at EA and suppression at LAF.

Depending on the NPY receptor subtypes and the target cells, various signaling pathways appear to be involved in the regulation of cell proliferation. NPY1R has been reported to enhance proliferation via $\mathrm{Wnt} / \beta$-catenin pathway in bone marrow stromal cells [30] and MEK signal in adipocyte precursor cells [7]. NPY5R is 
A

\section{Early antral follicles}

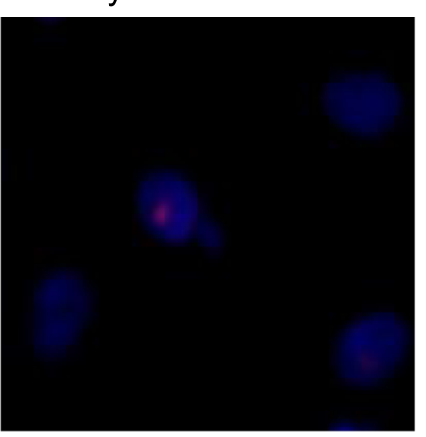

CTL

NPY

(0.1 nM)
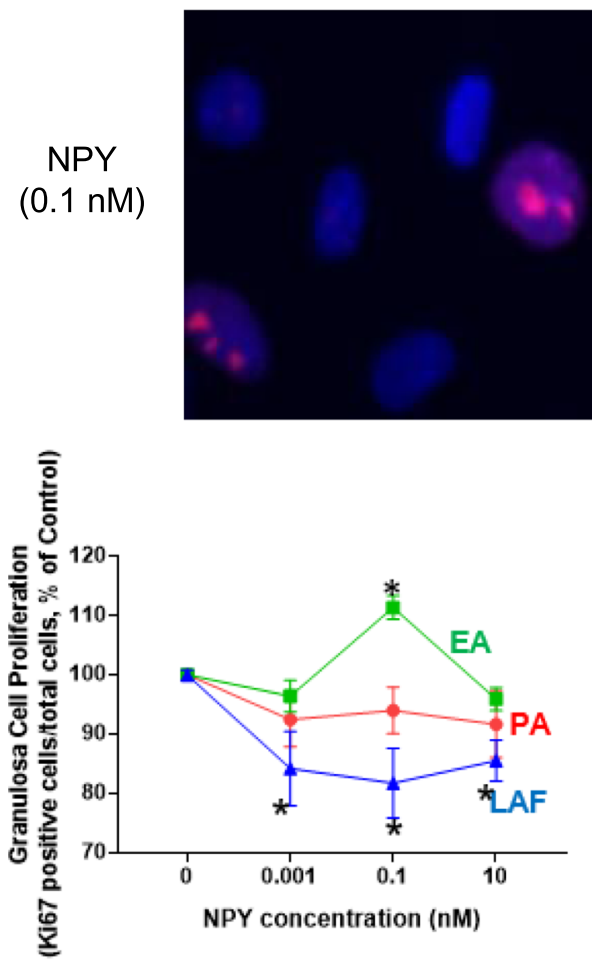

C

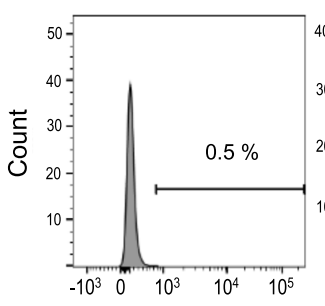

Negative control
B
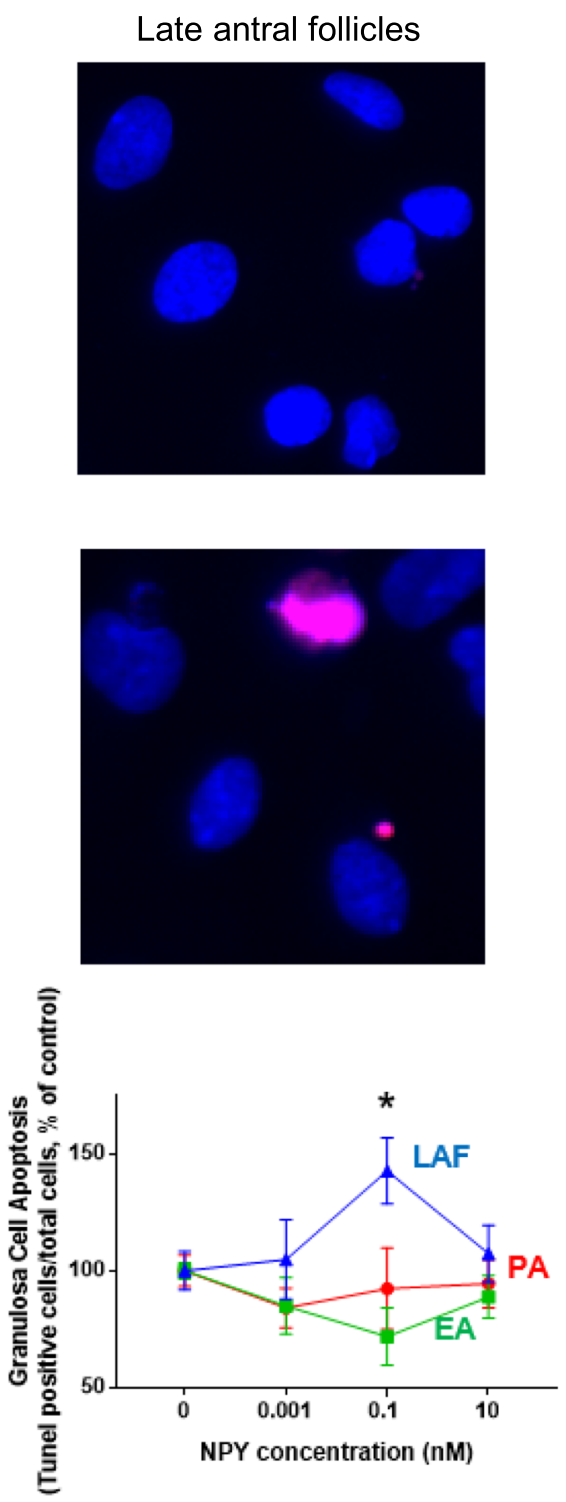

NPY $(0.1 \mathrm{nM})$

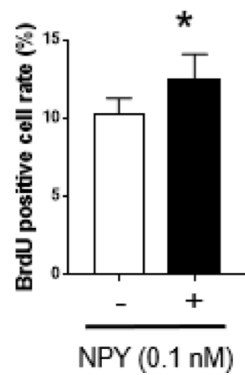

Fig. 4 (See legend on next page.) 
(See figure on previous page.)

Fig. 4 NPY induces granulosa cell proliferation in EA and reduced that in LAF. Granulosa cells of PA, EA and LAF were isolated separately using rats primed with eCG, as described in the methods section. $\mathbf{a}, \mathbf{b}$ Granulosa cells were incubated with various concentration of NPY for $24 \mathrm{~h}$ and immunofluorescence of Ki67 (proliferation) and TUNEL assay (apoptosis) were performed. NPY induced Ki67-positivity of granulosa cells in EA (0.1 $\mathrm{nM})$, while reduced that in LAF $(0.001,0.1,10 \mathrm{nM} ; A)$. NPY induced TUNEL-positivity of granulosa cells in LAF (0.1 nM; B). c Granulosa cells from EA were incubated with NPY $(0.1 \mathrm{nM})$ for $24 \mathrm{~h}$ and BrdU $(10 \mu \mathrm{M})$ for last $4 \mathrm{~h}$, and BrdU positive cells were detected by flow cytometry. Numbers displayed indicate percentage of single BrdU positive cells. NPY (0.1 nM) induced BrdU-positive granulosa cells in EA. These results suggest that NPY regulates granulosa cell proliferation and apoptosis in a follicular stage-dependent manner, inducing granulosa cell proliferation in early antral stage but apoptosis in late antral stage. Results are expressed as mean \pm SEM of independent replicates $(A, n=5 ; B, n=4 ; C, n=4)$ and analyzed by two-way ANOVA and Tukey's multiple comparison $\mathbf{a}, \mathbf{b}$ and paired t-test $\mathbf{c}$. ${ }^{*}, p<0.05$ (vs. without NPY). Representative pictures and flow cytometry results are shown

known to induce proliferation via MEK in cardiomyocytes [31], bone marrow stromal cells [24] and breast cancer cells [28], and STAT3 in vascular smooth muscle cells [23]. It has been reported that MEK and AKT signal pathways were important of proliferation in granulosa cells $[32,33]$. Support for this notion came from the observation that the MEK inhibitor U0126 attenuates hepatocyte growth factor-induced proliferation in human granulosa-like tumor cell line (KGN cells) [32] and insulin-like growth factor 1-induced, AKT-mediated proliferation in human luteinized granulosa cells [33]. In the present studies, we have demonstrated that NPYinduced proliferation in granulosa cells may involve NPY5R and the MEK signal pathway. Although NPY5Rmutant female mice are fertile and NPY has been suggested to be a factor which regulates granulosa cell proliferation and follicle selection [34].

\section{Conclusions}

We have demonstrated for the first time the possible role of NPY on ovarian folliculogenesis. We propose the following hypothesis (Fig. 6): during the preantral follicle development, granulosa cells express minimal NPY and NPY5R, and NPY does not affect proliferation nor apoptosis. As the follicle emerges in the early antral stage, granulosa cells express a greater amount of NPY, which induces the proliferation of granulosa cells in an autocrine and a NPY5R-dependent manner. However, during late antral development, NPY and NPY5R expression in granulosa cells decreases, resulting in reduction of proliferation and induction of apoptosis. Whether NPY plays a role in pathogenesis of follicular developmental disorders (e.g. polycystic ovarian syndrome) remains to be determined.

\section{Methods}

\section{Animal}

Female SD rats were obtained from Charles River Canada (Montreal, QC, Canada). All animal procedures were carried out in accordance with the Guidelines for the Care and Use of Laboratory Animals and the Canadian Council on Animal Care, and approved by the
University of Ottawa Animal Care Committee (Protocol \# OHRI-1624).

\section{Reagents and materials}

Folligon (eCG, equine Chorionic Gonadotropin) was purchased from Merck (Kirkland, Canada). RNeasy minikit and primers were purchased from QIAGEN (Hilden, Germany). SYBER Green I Master, cOmplete (a proteinase inhibitor), PhosSTOP (a phosphatase inhibitor cocktail) and In Situ Cell Death Detection Kit were obtained from Roche Diagnostics GmbH (Mannheim, Germany). Rat Neuropeptide $Y$ was purchased from Abcam (Cambridge, MA). Bovine serum albumin (BSA), paraformaldehyde (PFA), phosphate-buffered saline containing $0.05 \%$ tween-20 (PBS-T) and 5-bromo-2-deoxyuridine $(\mathrm{BrdU})$ were purchased from Millipore Sigma (Oakville, Canada). Six-well plate and 8 chamber slides were from Corning (Corning, NY). High-Capacity cDNA Reverse Transcription Kits, M199, penicillin and streptomycin, amphotericin B, fetal bovine serum (FBS) and SlowFade $^{\mathrm{Tm}}$ Gold Antifade Mountant with DAPI were purchased from Thermo Fisher Scientific (Waltham, MA). Sodium citrate was purchased from Fisher Scientific (Nepean, Canada). Cell lysis buffer was purchased from Cell signaling technology (Danvers, MA). Bio-Rad DC Protein Assay Reagent was from Bio-Rad Laboratories (Hercules, CA). The antibodies used in this study are described in Additional file 1: Table S1.

\section{Immunofluorescence}

For immunofluorescence of NPY, whole ovaries from 21-day-old immature rats were fixed in $4 \%$ PFA (24 h, $4{ }^{\circ} \mathrm{C}$ ) and embedded in paraffin. Thirty serial sections (Thickness: $5 \mu \mathrm{m}$ ) were cut from each sample to attain $150 \mu \mathrm{m}$ of distance between analyzed sections and to avoid the double analysis of same follicle [35]. Ovarian sections were deparaffinized and hydrated. Antigen retrieval was achieved with citrate buffer (10 mM; pH 6.0, boiled by microwave, $1 \mathrm{~min}$ ). Sections were washed in PBS-T and non-specific bindings were blocked with 5\% skim milk [40 min, room temperature (RT)]. The slides were incubated with rabbit anti-NPY or rabbit IgG, polyclonal (isotype control; overnight, $4{ }^{\circ} \mathrm{C}$ ) followed by anti- 


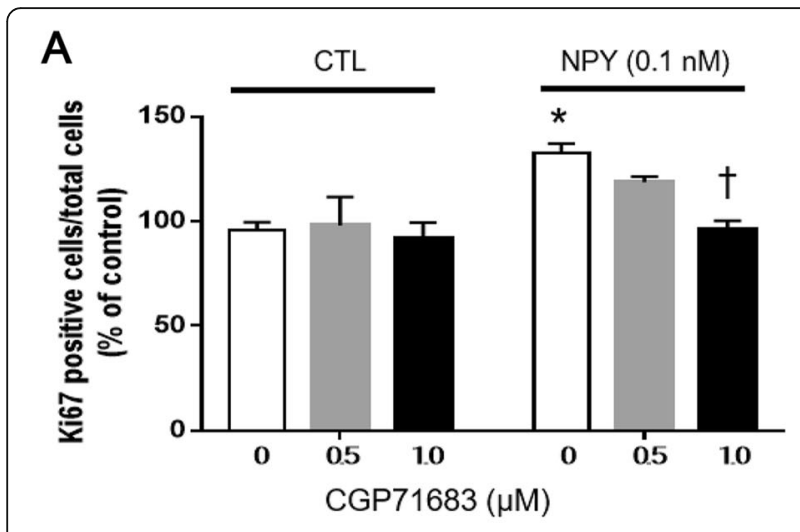

B

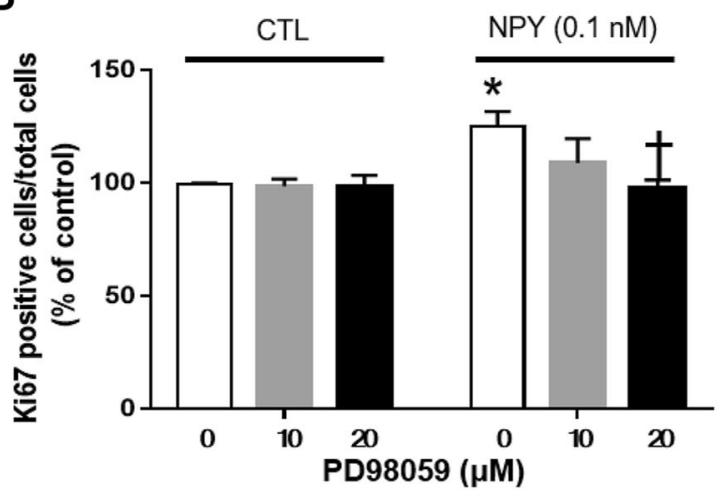

C

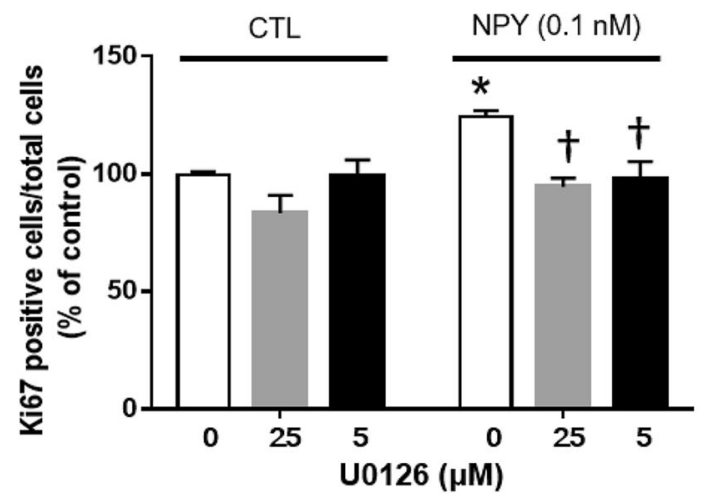

Fig. 5 CGP71683, PD98059 and U0126 inhibited NPY-induced granulosa cell proliferation in early antral follicles. Granulosa cells in EA were pre-incubated with CGP71683 (NPY5R antagonist), PD98059 or U0126 (MEK inhibitors) for $1 \mathrm{~h}$ and stimulated with NPY (0.1 nM) for $24 \mathrm{~h}$. Cell proliferation (Ki67-positivity) was assessed by immunocytochemistry. CGP71683, PD98059 or U0126 inhibited NPYinduced Ki67-positivity of granulosa cells. Each granulosa cell replicate was isolated from at least two rats. Results are expressed as mean \pm SEM of independent replicates $(A, n=4 ; B, n=5 ; C, n=4)$ and analyzed by two-way ANOVA and Tukey's multiple comparison. *, $p<0.05$ (vs. without NPY and CGP71683/MEK inhibitors); $\dagger, p<$ 0.05 (vs. with NPY) rabbit conjugated with alexa-fluor 594 (1 h, RT). Ovarian sections were mounted with SlowFade ${ }^{\mathrm{rm}}$ Gold Antifade Mounting with DAPI, and images were observed by fluorescence microcopy (Zeiss Axioplan 2, Zeiss, North York, Canada), recorded with the Axion Vision program (Axion Vision software, Zeiss) and analyzed using FIJI software.

Immunofluorescence of Ki67 was performed to assess granulosa cell proliferation [36]. Cells were fixed with 4\% PFA (60 min, RT) and treated with $0.25 \%$ Triton- 100 (3 min, RT). Nonspecific binding was blocked with 3\% BSA (30 min, RT). Cells were incubated (overnight, $4{ }^{\circ} \mathrm{C}$ ) with anti-Ki67 antibody or rabbit IgG, polyclonal (isotype control) followed by anti-rabbit IgG conjugated with alexa-fluor 594 (1 h, RT). Cells were washed, mounted with SlowFade ${ }^{\mathrm{mm}}$ Gold Antifade Mountant with DAPI and observed by fluorescence microscope with images recorded using the Axion Vision program. At least 400 cells were observed per experimental group.

\section{Granulosa cell isolation}

Granulosa cells from follicles at different stages of development [37] were isolated as previously described [38]. Briefly, ovaries from 21-day-old rats injected with eCG (10 IU, ip) were collected at various period after injection and kept in M199: $0 \mathrm{~h}$ (predominantly PA and EA), $24 \mathrm{~h}$ (predominantly EA), and $48 \mathrm{~h}$ (predominantly LAF and preovulatory follicles). Granulosa cells were plated overnight $\left(0.5 \times 10^{6}\right.$ per well in 6 well plate; 35,000 per well in chamber slide) in M199 containing penicillin, streptomycin and amphotericin B with 10\% FBS under a humidified atmosphere of $95 \%$ air and $5 \% \mathrm{CO}_{2}$. After a 24 $\mathrm{h}$ culture in serum-reduced medium, granulosa cells were treated with NPY for $24 \mathrm{~h}$ for assessment of Ki67 positivity, TUNEL and BrdU assays.

\section{Western blotting}

Granulosa cells were lysed in cell lysis buffer containing cOmplete and PhosSTOP, a protease inhibitor and phosphatase inhibitor cocktail. Following sonication, the lysates were centrifuged $\left(20 \mathrm{~min}, 15,000 \mathrm{~g}, 4{ }^{\circ} \mathrm{C}\right)$ and protein concentration was determined (Bio-Rad DC Protein Assay Reagent). To analyze NPY and NPY receptors contents, $40 \mu \mathrm{g}$ and $20 \mu \mathrm{g}$ protein lysates were separated by $16.5 \%$ Tricine-SDS-PAGE and 10\% SDS-PAGE, respectively. Separated proteins were electro-transferred to PVDF membranes (for NPY) and nitrocellulose membranes (for NPY receptors). Nonspecific bindings to the membranes were blocked with skim milk $(5 \%, 1 \mathrm{~h}, \mathrm{RT})$. Blots were incubated with primary antibody (overnight; $4{ }^{\circ} \mathrm{C}$ ) and then with HRP-conjugated secondary antibody $(1 \mathrm{~h}, \mathrm{RT})$. Peroxidase activity was visualized with an enhanced chemiluminescence kit, and membranes were 


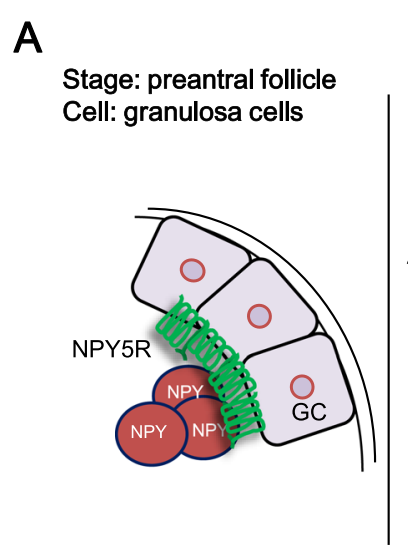

\section{B}

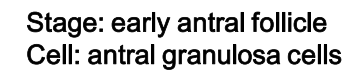

C Cell: antral granulosa cells
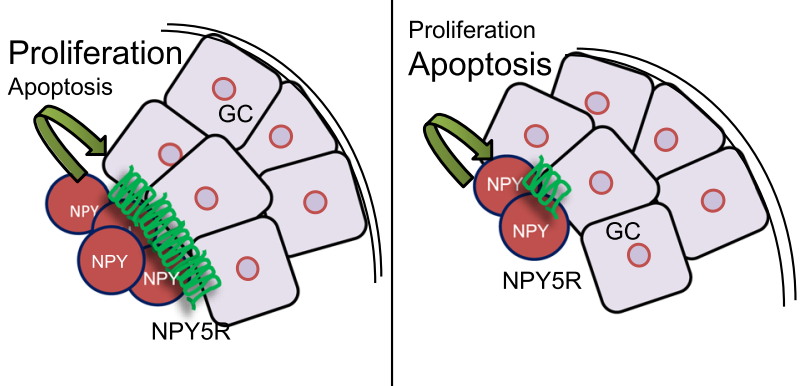

Fig. 6 A hypothetical model illustrating NPY function in granulosa cells during the follicular development. a At preantral follicles, NPY plays minimal or no role in the regulation of proliferation nor apoptosis in granulosa cells. b At early antral follicles, NPY-secreted by granulosa cells induces proliferation via NPY5R in an autocrine manner. c At late antral follicles, both of NPY-secreted by granulosa cells and granulosa cell NPY5R expression decrease comparing to those at early antral follicles. NPY-secreted by granulosa cells induced apoptosis in an autocrine manner, which may involve other NPY receptor

exposed to X-ray film. Signals were densitometrically quantified using FIJI software.

\section{RNA extraction, reverse transcription and real-time PCR}

RNA was extracted from granulosa cells by RNeasy minikit. One $\mu \mathrm{g}$ of total RNA was reverse transcribed in a 20- $\mu$ l of a reaction, using a High Capacity cDNA Reverse Transcription Kit. Real-time PCR was run using Light Cycler 480 SYBR Green I (Roche Diagnostics $\mathrm{GmbH})$ with the following conditions: Npy, 45 cycles at $95^{\circ} \mathrm{C}(10 \mathrm{~s}), 60^{\circ} \mathrm{C}(10 \mathrm{~s}), 72{ }^{\circ} \mathrm{C}(7 \mathrm{~s}) ;$ Gapdh, 45 cycles at $95^{\circ} \mathrm{C}(10 \mathrm{~s}), 60^{\circ} \mathrm{C}(10 \mathrm{~s}), 72^{\circ} \mathrm{C}(7 \mathrm{~s})$. Values were calculated by subtracting data for $\mathrm{Ct}$ values of the internal standard (glyceraldehyde dehydrogenase, Gapdh) from $\mathrm{Ct}$ values of Npy. Data were analyzed by the $2-\Delta \Delta \mathrm{CT}$ method.

\section{Apoptosis assay}

Apoptosis was assessed by the TUNEL assay, using the In Situ Cell Death Detection Kit. Stained cells were observed by fluorescence microcopy and images were recorded using the Axion Vision program. At least 400 cells were observed per experimental group.

\section{BrdU (5-bromo-2-deoxyuridine) incorporation}

Granulosa cells were incubated with BrdU $(10 \mu \mathrm{M}$, Millipore Sigma) for $4 \mathrm{~h}$, fixed with $70 \%$ ethanol and kept at $-20 \mathrm{C}^{\circ}$ overnight. They were incubated with $2 \mathrm{~N} \mathrm{HCl} /$ $0.5 \%$ TritonX-100 (30 min, RT) to produce singlestranded DNA and neutralized with $0.1 \mathrm{M}$ Sodium tetraborate decahydrate $(2 \mathrm{~min}, \mathrm{RT})$, and stained with antiBrdU-FITC antibody (30 min, RT). Flow cytometry acquisition was performed using BD LSRFortessaTM (BD, Franklin, NJ) and the data were analyzed using the
Flow $\mathrm{J}^{\circ}$ software. Doublets were excluded with forward scatter height against forward scatter area and subsequently side scatter height against side scatter area. Granulosa cells incubated without BrdU were used as a negative control.

\section{Statistical analysis}

Results are expressed as mean \pm SEM and analyzed by paired $t$ test, unpaired $t$ test or one- or two-way ANOVA, with Tukey's post hoc analysis for multiple comparisons. Statistical analyses were performed using Prism 7 (GraphPad software Inc.). Significant differences were considered at $P<0.05$.

\section{Supplementary information}

Supplementary information accompanies this paper at https://doi.org/10. 1186/s13048-019-0608-z.

Additional file 1: Table S1. Antibodies used in the present studies.

\section{Abbreviations}

BAX: BCl-2-associated X; BrdU: 5-bromo-2-deoxyuridine; BSA: Bovine serum albumin; EA: Early antral follicles; eCG: Equine chorionic gonadotropin; FBS: Fetal bovine serum; LAF: Late antral follicles; MEK: Mitogen-activated protein kinase; NPY: Neuropepetide Y; NPY1R: NPY receptor Y1; NPY2R: NPY receptor Y2; NPY4R: NPY receptor Y4; NPY5R: NPY receptor Y5; PA: Preantral follicles; PBS-T: Phosphate-buffered saline containing 0.05\% tween-20; PCNA: Proliferating cell nuclear antigen; PCR: Polymerase chain reaction; PFA: Paraformaldehyde; RT: Room temperature; SD rat: Sprague Dawley rat

\section{Acknowledgements}

Not applicable.

\section{Authors' contributions}

YU developed the study, designed and performed the experiments, interpreted the results and wrote the manuscript; RS helped in statistical analysis, the design of experiments and review and modified the manuscript; PDAL helped in establishing the experimental protocols, the design of experiments and review and modified the manuscript; YO helped the design 
of experiments and review and modified the manuscript; BKT provided the research funding, developed the study, designed the experiments and review and modified the manuscript. All authors read and approved the final manuscript.

\section{Funding}

This work was supported by Canadian Institutes of Health Research; and Kanzawa medical research foundation, Overseas study grant.

\section{Availability of data and materials}

All data is contained in the manuscript.

\section{Ethics approval and consent to participate}

All animal procedures were carried out in accordance with the Guidelines for the Care and Use of Laboratory Animals and the Canadian Council on Animal Care, and approved by the University of Ottawa Animal Care Committee (Protocol \# OHRI-1624).

\section{Consent for publication}

Not applicable.

\section{Competing interests}

All authors declare that they have no competing interests.

\section{Author details}

${ }^{1}$ Departments of Obstetrics \& Gynecology and Cellular \& Molecular Medicine, Interdisciplinary School of Health Sciences, University of Ottawa; Chronic Disease Program, Ottawa Hospital Research Institute, Critical Care Wing, 3rd floor, Room W3107, 501 Smyth Road, Ottawa, ON K1H 8L6, Canada. 2Department of Obstetrics and Gynecology, the University of Tokyo, 7-3-1 Hongo, Bunkyo-ku, Tokyo 113-8655, Japan. ${ }^{3}$ Queen's Cardiopulmonary Unit, Queen's University, BioSciences Complex, Room 1605, 116 Barrie Street, Kingston, ON K7L 3N6, Canada.

\section{Received: 18 October 2019 Accepted: 29 December 2019}

\section{Published online: 08 January 2020}

\section{References}

1. Tatemoto K, Carlquist M, Mutt V. Neuropeptide Y--a novel brain peptide with structural similarities to peptide $Y Y$ and pancreatic polypeptide. Nature. 1982:296(5858):659-60.

2. Gehlert DR. Introduction to the reviews on neuropeptide Y. Neuropeptides. 2004;38(4):135-40.

3. Wu Q, Boyle MP, Palmiter RD. Loss of GABAergic signaling by AgRP neurons to the parabrachial nucleus leads to starvation. Cell. 2009;137(7):1225-34.

4. Kim TT, Dyck JR. Is AMPK the savior of the failing heart? Trends Endocrinol Metab. 2015;26(1):40-8.

5. Gotzsche CR, Woldbye DP. The role of NPY in learning and memory. Neuropeptides. 2016:55:79-89.

6. Muroi $Y$, Ishii T. A novel neuropeptide $Y$ neuronal pathway linking energy state and reproductive behavior. Neuropeptides. 2016;59:1-8.

7. Yang $K$, Guan H, Arany E, Hill DJ, Cao X. Neuropeptide $Y$ is produced in visceral adipose tissue and promotes proliferation of adipocyte precursor cells via the Y1 receptor. FASEB J. 2008;22(7):2452-64.

8. Keator CS, Custer EE, Hoagland TA, Schreiber DT, Mah K, Lawson AM, et al. Evidence for a potential role of neuropeptide $Y$ in ovine corpus luteum function. Domest Anim Endocrinol. 2010;38(2):103-14.

9. McDonald JK, Dees WL, Ahmed CE, Noe BD, Ojeda SR. Biochemical and immunocytochemical characterization of neuropeptide $Y$ in the immature rat ovary. Endocrinol. 1987;120(5):1703-10.

10. Jorgensen JC. Neuropeptide $Y$ in mammalian genital tract: localization and biological action. Dan Med Bull. 1994;41(3):294-305.

11. Kanzaki M, Fujisawa M, Okuda Y, Okada H, Arakawa S, Kamidono S. Expression and regulation of neuropeptide $Y$ messenger ribonucleic acid in cultured immature rat Leydig and Sertoli cells. Endocrinol. 1996;137(4):1249-57.

12. Pitzel $L$, Jarry $H$, Wuttke $W$. Effects of substance-P and neuropeptide-Y on in vitro steroid release by porcine granulosa and luteal cells. Endocrinol. 1991;129(2):1059-65.

13. Barreca A, Valli B, Cesarone A, Arvigo M, Balasini M, La B, Sala G, et al. Effects of the neuropeptide $Y$ on estradiol and progesterone secretion by human granulosa cells in culture. Fertil Steril. 1998;70(2):320-5.
14. Sirotkin AV, Kardosova D, Alwasel SH, Harrath AH. Neuropeptide Y directly affects ovarian cell proliferation and apoptosis. Reprod Biol. 2015;15(4):257-60.

15. Foradori CD, Whitlock BK, Daniel JA, Zimmerman AD, Jones MA, Read CC, et al. Kisspeptin stimulates growth hormone release by utilizing neuropeptide $Y$ pathways and is dependent on the presence of ghrelin in the ewe. Endocrinol. 2017;158(10):3526-39.

16. Wojcik-Gladysz A, Polkowska J. Neuropeptide Y--a neuromodulatory link between nutrition and reproduction at the central nervous system level. Reprod Biol. 2006;6(Suppl 2):21-8

17. Barb CR, Kraeling RR, Rampacek GB, Hausman GJ. The role of neuropeptide $Y$ and interaction with leptin in regulating feed intake and luteinizing hormone and growth hormone secretion in the pig. Reprod. 2006;131(6):1127-35.

18. Khorram O, Pau KY, Spies HG. Release of hypothalamic neuropeptide $Y$ and effects of exogenous NPY on the release of hypothalamic GnRH and pituitary gonadotropins in intact and ovariectomized does in vitro. Pept. 1988;9(2):411-7.

19. Pinilla L, Fernandez-Fernandez R, Roa J, Castellano JM, Tena-Sempere M, Aguilar E. Selective role of neuropeptide $Y$ receptor subtype $Y 2$ in the control of gonadotropin secretion in the rat. Am J Physiol Endocrinol Metab. 2007;293(5):E1385-92.

20. Di Yorio MP, Bilbao MG, Faletti AG. Neuropeptide $Y$ regulates the leptin receptors in rat hypothalamic and pituitary explant cultures. Regul Pept. 2014;188:13-20.

21. Siawrys $\mathrm{G}$, Buchowski $\mathrm{H}$. Modulation of anterior pituitary cell luteinizing hormone secretory activity by neuropeptide $Y$ in early pregnant pigs. J Physiol Pharmacol. 2018;69(5):719-726.

22. Ding $X$, Kou $X$, Zhang $Y$, Zhang $X$, Cheng $G$, Jia T. Leptin siRNA promotes ovarian granulosa cell apoptosis and affects steroidogenesis by increasing NPY2 receptor expression. Gene. 2017;633:28-34.

23. Zhang P, Qi YX, Yao QP, Chen XH, Wang GL, Shen BR, et al. Neuropeptide $Y$ stimulates proliferation and migration of vascular smooth muscle cells from pregnancy hypertensive rats via Y1 and Y5 receptors. PLoS One. 2015;10(7): e0131124.

24. Igura K, Haider H, Ahmed RP, Sheriff S, Ashraf M. Neuropeptide y and neuropeptide y y 5 receptor interaction restores impaired growth potential of aging bone marrow stromal cells. Rejuvenation Res. 2011;14(4):393-403.

25. Meng D, Wu T, Rao U, North CS, Xiao H, Javors MA, et al. Serum NPY and BNDF response to a behavioral stressor in alcohol-dependent and healthy control participants. Psychopharmacol. 2011;218(1):59-67.

26. Panuccio V, Cutrupi S, Pizzini P, Mallamaci F, Tripepi G, Zoccali C. Neuropeptide $Y$ and markers of osteoblast activity in dialysis patients: a cross-sectional study. Am J Kidney Dis. 2007;50(6):1001-8.

27. Kitlinska J. Neuropeptide Y (NPY) in neuroblastoma: effect on growth and vascularization. Pept. 2007;28(2):405-12.

28. Medeiros PJ, Al-Khazraji BK, Novielli NM, Postovit LM, Chambers AF, Jackson DN. Neuropeptide $Y$ stimulates proliferation and migration in the $4 \mathrm{~T} 1$ breast cancer cell line. Int J Cancer. 2012;131(2):276-86.

29. Sheriff S, Ali M, Yahya A, Haider KH, Balasubramaniam A, Amlal H. Neuropeptide $Y$ Y5 receptor promotes cell growth through extracellular signal-regulated kinase signaling and cyclic AMP inhibition in a human breast cancer cell line. Mol Cancer Res. 2010;8(4):604-14.

30. Wu J, Liu S, Meng H, Qu T, Fu S, Wang Z, et al. Neuropeptide Y enhances proliferation and prevents apoptosis in rat bone marrow stromal cells in association with activation of the Wnt/beta-catenin pathway in vitro. Stem Cell Res. 2017;21:74-84.

31. Pellieux C, Sauthier T, Domenighetti A, Marsh DJ, Palmiter RD, Brunner HR, et al. Neuropeptide Y (NPY) potentiates phenylephrine-induced mitogenactivated protein kinase activation in primary cardiomyocytes via NPY Y5 receptors. Proc Natl Acad Sci U S A. 2000;97(4):1595-600.

32. Taniguchi F, Harada T, Deura I, Iwabe T, Tsukihara S, Terakawa N. Hepatocyte growth factor promotes cell proliferation and inhibits progesterone secretion via PKA and MAPK pathways in a human granulosa cell line. Mol Reprod Dev. 2004;68(3):335-44

33. Goto M, Iwase A, Harata T, Takigawa S, Suzuki K, Manabe S, et al. IGF1induced AKT phosphorylation and cell proliferation are suppressed with the increase in PTEN during luteinization in human granulosa cells. Reprod. 2009;137(5):835-42.

34. Marsh DJ, Hollopeter G, Kafer KE, Palmiter RD. Role of the $Y 5$ neuropeptide $Y$ receptor in feeding and obesity. Nat Med. 1998;4(6):718-21.

35. Lima PDA, Nivet AL, Wang Q, Chen YA, Leader A, Cheung A, et al. Polycystic ovary syndrome: possible involvement of androgen-induced, chemerin- 
mediated ovarian recruitment of monocytes/macrophages. Biol Reprod. 2018;99(4):838-52.

36. Scholzen T, Gerdes J. The Ki-67 protein: from the known and the unknown. J Cell Physiol. 2000;182(3):311-22.

37. Wang H, Jiang JY, Zhu C, Peng C, Tsang BK. Role and regulation of nodal/ activin receptor-like kinase 7 signaling pathway in the control of ovarian follicular atresia. Mol Endocrinol. 2006;20(10):2469-82.

38. Wang Q, Kim JY, Xue K, Liu JY, Leader A, Tsang BK. Chemerin, a novel regulator of follicular steroidogenesis and its potential involvement in polycystic ovarian syndrome. Endocrinol. 2012;153(11):5600-11.

\section{Publisher's Note}

Springer Nature remains neutral with regard to jurisdictional claims in published maps and institutional affiliations.

Ready to submit your research? Choose BMC and benefit from:

- fast, convenient online submission

- thorough peer review by experienced researchers in your field

- rapid publication on acceptance

- support for research data, including large and complex data types

- gold Open Access which fosters wider collaboration and increased citations

- maximum visibility for your research: over $100 \mathrm{M}$ website views per year

At BMC, research is always in progress.

Learn more biomedcentral.com/submissions 